\title{
SMALL SCALE EQUIDISTRIBUTION FOR A POINT SCATTERER ON THE TORUS
}

\author{
NADAV YESHA
}

\begin{abstract}
We study the small scale distribution of the eigenfunctions of a point scatterer (the Laplacian perturbed by a delta potential) on two- and three-dimensional flat tori. In two dimensions, we establish small scale equidistribution for the "new" eigenfunctions holding all the way down to the Planck scale. In three dimensions, small scale equidistribution is established for all of the "new" eigenfunctions at certain scales.
\end{abstract}

\section{INTRODUCTION}

1.1. Background. One of the main goals in the field of Quantum Chaos is understanding the distribution of quantum eigenstates in the semiclassical limit. For example, the celebrated Quantum Ergodicity Theorem ("Shnirelman's Theorem") [26, 32, 5] asserts that if the underlying classical dynamics of a quantum system is ergodic, then almost all eigenstates are equidistributed in phase space. In particular, let $\mathcal{M}$ be a smooth, compact Riemannian manifold, and let $\left\{\phi_{j}\right\}$ be an orthonormal basis of $L^{2}(\mathcal{M}, d \mathrm{vol})$ consisting of Laplace eigenfunctions with corresponding eigenvalues $\left\{E_{j}\right\}$ (where $d \mathrm{vol}$ is the normalized Riemannian volume form). If the geodesic flow on $\mathcal{M}$ is ergodic, then there exists a density one subsequence $\left\{\phi_{j_{k}}\right\}$, such that for every "nice" $\mathcal{A} \subseteq \mathcal{M}$, we have

$$
\int_{\mathcal{A}}\left|\phi_{j_{k}}(y)\right|^{2} d \operatorname{vol}(y) \sim \operatorname{vol}(\mathcal{A}) \quad(k \rightarrow \infty) .
$$

Moreover, it is expected [1, 2] that generically equidistribution should hold at smaller scales, i.e., when $\mathcal{A}=B_{r}(x)$ is the radius $r$ geodesic ball centred at $x$, and $r$ decays slower than the Planck scale $E_{j}^{-1 / 2}$.

In this paper, we study small scale equidistribution for a point scatterer, or the Laplacian perturbed with a delta potential on the flat torus $\mathbb{T}^{d}=\mathbb{R}^{d} / 2 \pi \mathbb{Z}^{d}(d=2,3)$, an important model in Quantum Chaos for studying the transition between chaos and integrability. The underlying classical dynamics of a toral point scatterer is integrable, since it is identical to the geodesic flow on the torus (excluding a measure zero set of trajectories); on the other hand, numerical experiments suggest that the

\footnotetext{
${ }^{1}$ The normalization by $2 \pi$ is introduced to facilitate the notation below.
} 
eigenfunctions and spectrum of this system display chaotic features such as Gaussianlike value distribution and level repulsion (see [23]).

1.2. Small scale equidistribution. Small scale equidistribution is a very active area of research nowadays, though most of the results are partial. On the modular surface, Luo and Sarnak [16] established (1.1) with balls $\mathcal{A}=B_{r}(x)$ of radii $r>E_{j_{k}}^{-\alpha}$ (for some small $\alpha>0$ ) for a density one sequence of Hecke-Maass forms; Young [17] showed that under the Generalized Riemann Hypothesis, (1.1) holds with balls $\mathcal{A}=B_{r}(x)$ of radii $r>E_{j}^{-1 / 6}$ for all such forms. Hezari and Rivière [12] and Han [8] established (a non-uniform version of) (1.1) with balls $\mathcal{A}=B_{r}(x)$ of radii $r>\left(\log E_{j_{k}}\right)^{-\alpha}$ on compact negatively curved manifolds. Further results are due to Han [9] (small scale equidistribution for random eigenbases on a certain class of "symmetric" manifolds), Han and Tacy [10] (random combinations of Laplace eigenfunctions on compact manifolds), Humphries [14] (small scale equidistribution for Hecke-Maass forms, with balls $\mathcal{A}=B_{r}(x)$ whose centres are random. See also [7, 28] for results on the torus), and de Courcy-Ireland [6] (discrepancy estimates for random spherical harmonics).

An example of a manifold with small scale equidistribution holding (almost) all the way down to Planck scale is the the two-dimensional torus $\mathbb{T}^{2}$, as was demonstrated by Lester and Rudnick [21] (see also Hezari and Rivière [13]), who showed that for every orthonormal basis $\left\{\phi_{j}\right\}$ of toral Laplace eigenfunctions, there exists a density one subsequence $\left\{\phi_{j_{k}}\right\}$ such that

$$
\int_{B_{r}(x)}\left|\phi_{j_{k}}(y)\right|^{2} d \operatorname{vol}(y) \sim \operatorname{vol}\left(B_{r}(x)\right) \quad(k \rightarrow \infty)
$$

uniformly for $r>E_{j_{k}}^{-1 / 2+o(1)}$ and $x \in \mathbb{T}^{2}$. More generally, for the $d$-dimensional torus $\mathbb{T}^{d}$, they established (1.2) for a density one sequence of toral Laplace eigenfunctions uniformly for $r>E_{j_{k}}^{-\frac{1}{2(d-1)}+o(1)}$ and $x \in \mathbb{T}^{d}$, and proved that the lower bound on the radii is sharp (a refined version of Lester and Rudnick's result for the two-dimensional torus was obtained by Granville and Wigman [7] more recently).

1.3. Toral point scatterers. A point scatterer on the torus is formally defined as the rank one singular perturbation

$$
-\Delta+\alpha\left\langle\delta_{x_{0}}, \cdot\right\rangle \delta_{x_{0}}
$$

where $\Delta$ is the Laplace-Beltrami operator, $\alpha \in \mathbb{R}$ is a coupling parameter, and $\delta_{x_{0}}$ is the Dirac delta potential at $x_{0} \in \mathbb{T}^{d}$. Rigorously, as described in [4], the operator (1.3) is realized as a self-adjoint operator acting on $L^{2}\left(\mathbb{T}^{d}\right)$ via the theory of self-adjoint extensions. One begins with the Laplacian acting on the domain of smooth functions vanishing near $x_{0}$; there exists a one parameter family of self-adjoint extensions of this 
operator denoted by $\Delta_{\phi}$ with $\phi \in(-\pi, \pi]$, each corresponding to an operator (1.3) with a particular value of $\alpha$ ( $\phi=\pi$ gives the trivial extension $\Delta_{\pi}=\Delta$ corresponding to $\alpha=0$, the only extension for manifolds of dimension $d \geq 4$ ).

The spectrum of a non-trivial self-adjoint extension consists of two types of eigenvalues:

- The "old", or unperturbed eigenvalues - these are the nonzero Laplace eigenvalues with multiplicities reduced by 1 . The corresponding eigenfunctions are precisely the Laplace eigenfunctions vanishing at $x_{0}$.

- A set $\Lambda=\Lambda_{\phi}$ of "new" or "perturbed" eigenvalues, of multiplicity 1 each, interlacing with the Laplace eigenvalues, so that there is a unique new eigenvalue between every two Laplace eigenvalues (its exact position depends on the choice of the self-adjoint extension). For a new eigenvalue $\lambda \in \Lambda$, the corresponding $L^{2}$-normalized new eigenfunction is $g_{\lambda}=G_{\lambda} /\left\|G_{\lambda}\right\|_{2}$, where $G_{\lambda}$ is Green's function $G_{\lambda}\left(x ; x_{0}\right)=(\Delta+\lambda)^{-1} \delta_{x_{0}}$.

The semiclassical limits of the new eigenfunctions of point scatterers on flat tori have been extensively studied in recent years (for a survey on some of the results, see [27]). Rudnick and Ueberschär [22] showed that for a point scatterer on a two-dimensional torus, a density one subsequence of the new eigenfunctions are equidistributed in configuration space, i.e., (1.1) holds along a density one subset $\Lambda^{\prime} \subseteq \Lambda$; by a density one subset we mean that

$$
\lim _{X \rightarrow \infty} \frac{\#\left\{\lambda \in \Lambda^{\prime}: \lambda \leq X\right\}}{\#\{\lambda \in \Lambda: \lambda \leq X\}}=1 .
$$

In [29], it was shown that for a point scatterer on the standard three-dimensional torus $\mathbb{T}^{3}$, equidistribution in configuration space holds for all of the new eigenfunctions (and along a density one subsequence of the new eigenfunctions for point scatterers on tori with a Diophantine aspect ratio). Recently, we were able to establish equidistribution in configuration space for tori with two point scatterers [31].

Equidistribution in full phase space (along a density one subsequence) was established both on the standard two-dimensional torus $\mathbb{T}^{2}$ by Kurlberg and Ueberschär [19], and on the standard three-dimensional torus $\mathbb{T}^{3}$ [30]. The quantum limits of a point scatterer on a torus with an irrational aspect ratio (also known as the Šeba billiard [23]) were further studied by Kurlberg-Ueberschär [20], who proved the existence of "scars", i.e., localized quantum limits. The existence of scars for arithmetic point scatterers was established by Kurlberg and Rosenzweig [18].

1.4. Statement of the main results. We now state our main results concerning small scale equidistribution for the new eigenfunctions $g_{\lambda}$ of toral point scatterers. We only discuss the standard flat two- and three-dimensional tori, although using our arguments, analogous (albeit weaker) results may also be obtained for any torus of 
the form $\mathbb{T}_{\mathcal{L}_{0}}^{2}=\mathbb{R}^{2} / 2 \pi \mathcal{L}_{0}$ where $\mathcal{L}_{0}=\mathbb{Z}(1 / a, 0) \oplus \mathbb{Z}(0, a)$ is a unimodular lattice (as in [22]), and for three-dimensional tori with Diophantine aspect ratio (as in [29]). The first principal result asserts the small scale equidistribution for the new eigenfunctions of a point scatterer on the standard flat two-dimensional torus $\mathbb{T}^{2}$, holding (almost) all the way down to the Planck scale. In particular, we significantly strengthen the main result in [22] in this case.

Theorem 1.1. Let $d=2$, and fix $\phi \in(-\pi, \pi)$. There is a subset $\Lambda^{\prime} \subseteq \Lambda_{\phi}$ of density one, such that for every $\epsilon>0$,

$$
\sup _{\substack{r>\lambda^{-1 / 2+\epsilon} \\ x \in \mathbb{T}^{2}}}\left|\frac{\int_{B_{r}(x)}\left|g_{\lambda}(y)\right|^{2} d y}{\pi r^{2}}-1\right| \rightarrow 0
$$

as $\lambda \rightarrow \infty$ along $\Lambda^{\prime}$.

Next, we establish the small scale equidistribution for the new eigenfunctions of a point scatterer on the standard flat three-dimensional torus $\mathbb{T}^{3}$. For balls with radii $r>\lambda^{-1 / 12+o(1)}$, our statement will hold for all new eigenfunctions, improving upon the principal result in [29].

Theorem 1.2. Let $d=3$, and fix $\phi \in(-\pi, \pi)$. For every $\epsilon>0$,

$$
\sup _{\substack{r>\lambda^{-1 / 12+\epsilon} \\ x \in \mathbb{T}^{3}}}\left|\frac{\int_{B_{r}(x)}\left|g_{\lambda}(y)\right|^{2} d y}{\frac{4}{3} \pi r^{3}}-1\right| \rightarrow 0
$$

as $\lambda \rightarrow \infty$ along $\Lambda_{\phi}$.

If one is willing to exclude a thin set of energy levels, the exponent of the radii in the three-dimensional case can be improved from $-1 / 12+\epsilon$ to $-1 / 6+\epsilon$.

Theorem 1.3. Let $d=3$, and fix $\phi \in(-\pi, \pi)$. There is a subset $\Lambda^{\prime} \subseteq \Lambda_{\phi}$ of density one, such that for every $\epsilon>0$,

$$
\sup _{\substack{r>\lambda^{-1 / 6+\epsilon} \\ x \in \mathbb{T}^{3}}}\left|\frac{\int_{B_{r}(x)}\left|g_{\lambda}(y)\right|^{2} d y}{\frac{4}{3} \pi r^{3}}-1\right| \rightarrow 0
$$

as $\lambda \rightarrow \infty$ along $\Lambda^{\prime}$.

It would be interesting to determine whether equidistribution holds down to the Planck scale in dimension three, or in a more restricted range imposed by the arithmetic setting (compare with the case $d>2$ in [21]).

In proving theorems 1.1, 1.2, and 1.3, we exploit the interlacing property of the new eigenvalues in an essential way; however, we do not use any further information regarding the exact position of the new eigenvalues. Thus, all of our results can be 
easily formulated for $\lambda$-dependent sequence of $\phi$. This is of significance, since the in the physics literature one often considers self-adjoint extensions with $\phi$ varying with $\lambda$ ("strong coupling", see [25, 27]).

Acknowledgements. The author would like to express his gratitude to Z. Rudnick and I. Wigman for useful discussions and comments. The research leading to these results was partially supported by the European Research Council under the European Union's Seventh Framework Programme (FP7/2007-2013), ERC grant agreement n 335141.

\section{Point scatterers on the torus}

2.1. The spectrum of the toral Laplacian. Let $\mathbb{T}^{d}=\mathbb{R}^{d} / 2 \pi \mathbb{Z}^{d}(d=2,3)$ be the standard flat $d$-dimensional torus, and let $\Delta$ be the associated Laplace-Beltrami operator. The spectrum of $-\Delta$ is the collection of all numbers that can be expressed as a sum of $d$ squares, denoted by

$$
\mathcal{N}_{d}=\left\{0=n_{1}<n_{2}<\ldots\right\} .
$$

Recall that by Landau's Theorem,

$$
\#\left\{n \in \mathcal{N}_{2}: n \leq X\right\} \sim K \frac{X}{\sqrt{\log X}},
$$

where $K=2^{-1 / 2} \prod_{p \equiv 3(4)}\left(1-p^{-2}\right)^{-1 / 2}=0.764 \ldots$ is the Landau-Ramanujan constant. In three-dimensions, $n \in \mathcal{N}_{3}$ if and only if in the representation $n=4^{a} n_{1}$ with $4 \nmid n_{1}$, the number $n_{1}$ satisfies $n_{1} \not \equiv 7$ (8). Moreover, as $X \rightarrow \infty$,

$$
\#\left\{n \in \mathcal{N}_{3}: n \leq X\right\} \sim \frac{5}{6} X
$$

Let $r_{d}(n)$ be the number of representations of $n \in \mathcal{N}_{d}$ as a sum of $d$ squares. For $d=2$, it is well-known that

$$
r_{2}(n)=O_{\eta}\left(n^{\eta}\right)
$$

(in fact, (2.1) implies that on average, $r_{2}(n)$ is of order of magnitude $\sqrt{\log n}$ ). For $d=3$, Siegel's Theorem [24] implies that for $n=4^{a} n_{1}$ with $4 \nmid n_{1}$, we have

$$
n_{1}^{\frac{1}{2}-\eta} \ll_{\eta} r_{3}(n)=r_{3}\left(n_{1}\right) \ll_{\eta} n_{1}^{\frac{1}{2}+\eta} .
$$


2.2. Toral point scatterers. Let $x_{0} \in \mathbb{T}^{d}$. A point scatterer, formally defined in (1.3), can be rigorously realized via the following procedure: denote by $D_{0}=$ $C_{c}^{\infty}\left(\mathbb{T}^{d} \backslash\left\{x_{0}\right\}\right)$ the space of smooth functions supported away from the point $x_{0}$, and denote by $-\Delta_{0}=-\Delta_{\mid D_{0}}$ the Laplacian restricted to this domain. This is a symmetric operator with deficiency indices $(1,1)$, hence there is a one parameter family of self-adjoint extensions, which we denote by $-\Delta_{x_{0}, \phi}, \phi \in(-\pi, \pi]$. For $\phi \neq \pi$ (the extension $\phi=\pi$ retrieves the standard Laplacian), the spectrum of $-\Delta_{x_{0}, \phi}$ consists of two types of eigenvalues:

- The "old", nonzero Laplace eigenvalues $0 \neq n \in \mathcal{N}_{d}$, which correspond to the Laplace eigenfunctions vanishing at $x_{0}$.

- A set $\Lambda=\Lambda_{\phi}$ of "new" or "perturbed" eigenvalues, which are the solutions to the equation

$$
\sum_{\xi \in \mathbb{Z}^{d}}\left(\frac{1}{|\xi|^{2}-\lambda}-\frac{|\xi|^{2}}{|\xi|^{4}+1}\right)=c_{0} \tan \frac{\phi}{2}
$$

where

$$
c_{0}=\sum_{\xi \in \mathbb{Z}^{d}} \frac{1}{|\xi|^{4}+1} .
$$

Thus, the elements of $\Lambda$ interlace with the elements of $\mathcal{N}_{d}$, i.e., between every two elements $n_{k}, n_{k+1} \in \mathcal{N}_{d}$ there is a unique element of $\Lambda$, so we label $\Lambda=\left\{\lambda_{0}, \lambda_{1}, \lambda_{2}, \ldots\right\}$, where

$$
\lambda_{0}<n_{1}<\lambda_{1}<n_{2}<\lambda_{2}<n_{3}<\lambda_{3}<\ldots
$$

As mentioned above, all of our results will still hold for an arbitrary set $\Lambda$ whose elements interlace with the elements of $\mathcal{N}_{d}$. The corresponding eigenfunctions, which are our main objects of study, are multiples of Green's functions $(\Delta+\lambda)^{-1} \delta_{x_{0}}$, admitting the $L^{2}$ expansion

$$
G_{\lambda}\left(x ; x_{0}\right):=-(2 \pi)^{d / 2}(\Delta+\lambda)^{-1} \delta_{x_{0}}=\sum_{\xi \in \mathbb{Z}^{d}} \frac{e^{i\left\langle x-x_{0}, \xi\right\rangle}}{|\xi|^{2}-\lambda} .
$$

Let

$$
g_{\lambda}(x)=g_{\lambda}\left(x ; x_{0}\right)=\frac{G_{\lambda}\left(x ; x_{0}\right)}{\left\|G_{\lambda}\right\|_{2}}
$$

be the $L^{2}$-normalized eigenfunctions. 
We will also work with a truncated version of the Green's functions $G_{\lambda}$. Let $L=\lambda^{\delta}$, $0<\delta<1$, and define the truncated Green's function

$$
G_{\lambda, L}\left(x ; x_{0}\right)=\sum_{\substack{\left.\xi \in \mathbb{Z}^{d} \\|| \xi\right|^{2}-\lambda \mid<L}} \frac{e^{i\left\langle x-x_{0}, \xi\right\rangle}}{|\xi|^{2}-\lambda}
$$

and the $L^{2}$-normalized truncated Green's function

$$
g_{\lambda, L}(x)=g_{\lambda, L}\left(x ; x_{0}\right)=\frac{G_{\lambda, L}\left(x ; x_{0}\right)}{\left\|G_{\lambda, L}\right\|_{2}} .
$$

\section{Proof of Theorem $1.1(d=2)$}

3.1. Preliminary lemmas. We now take $d=2$, and recall the following lemma, proved in 22] for two-dimensional flat tori with more general aspect ratios, which shows that along a density one subsequence, the distance between consecutive elements in $\mathcal{N}_{2}$ is small, so that almost all $\lambda \in \Lambda$ are close to an element of $\mathcal{N}_{2}$, implying a lower bound on the $L^{2}$-norm of $G_{\lambda}$. Recall the labeling (2.4), so that given $\lambda \in \Lambda$, we can denote $\lambda=\lambda_{k}$, where

$$
\cdots<\lambda_{k-1}<n_{k}<\lambda_{k}<n_{k+1}<\lambda_{k+1}<\ldots
$$

Lemma 3.1 ([22, Lemma 2.1, Lemma 4.1]).

(1) Along a density one subsequence $\left\{n_{k_{j}}\right\} \subseteq \mathcal{N}_{2}$, the spacings of $\mathcal{N}_{2}$ satisfy

$$
n_{k_{j}+1}-n_{k_{j}} \ll_{\eta} n_{k_{j}}^{\eta}
$$

for every $\eta>0$.

(2) There is a subset $\Lambda^{\prime}$ of density one in $\Lambda$, such that for all $\lambda_{k_{j}} \in \Lambda^{\prime}$ and all $\eta>0$, we have

$$
n_{k_{j}+1}-n_{k_{j}} \ll_{\eta} \lambda_{k_{j}}^{\eta}
$$

(3) For all $\lambda \in \Lambda^{\prime}$ and all $\eta>0$, we have $\left\|G_{\lambda}\right\|_{2} \gg_{\eta} \lambda^{-\eta}$.

Lemma 3.2. There exists a density one subset $\Lambda^{\prime} \subseteq \Lambda$, such that for every $\eta>0$, $0<\delta<1$, we have

$$
\left\|g_{\lambda}-g_{\lambda, L}\right\|_{2}^{2}=O_{\eta}\left(\frac{\lambda^{\eta}}{L}\right)
$$

as $\lambda \rightarrow \infty$ along $\lambda \in \Lambda^{\prime}$, where $L=\lambda^{\delta}$. 
Proof. We have

$$
\begin{aligned}
\left\|g_{\lambda}-g_{\lambda, L}\right\|_{2} & =\frac{1}{\left\|G_{\lambda, L}\right\|_{2}\left\|G_{\lambda}\right\|_{2}}\|\| G_{\lambda, L}\left\|_{2} G_{\lambda}-\right\| G_{\lambda}\left\|_{2} G_{\lambda, L}\right\|_{2} \\
& \leq \frac{\left\|G_{\lambda}-G_{\lambda, L}\right\|_{2}}{\left\|G_{\lambda}\right\|_{2}}+\frac{1}{\left\|G_{\lambda}\right\|_{2}}\left|\left\|G_{\lambda, L}\right\|_{2}-\left\|G_{\lambda}\right\|_{2}\right| \\
& \leq 2 \frac{\left\|G_{\lambda}-G_{\lambda, L}\right\|_{2}}{\left\|G_{\lambda}\right\|_{2}}
\end{aligned}
$$

Now,

$$
\begin{aligned}
\left\|G_{\lambda}-G_{\lambda, L}\right\|_{2}^{2} & =\sum_{\left.|| \xi\right|^{2}-\lambda \mid \geq L} \frac{1}{\left(|\xi|^{2}-\lambda\right)^{2}}=\sum_{\substack{n \geq 0 \\
|n-\lambda| \geq L}} \frac{r_{2}(n)}{(n-\lambda)^{2}} \\
& \ll_{\eta} \lambda^{\eta} \sum_{|n-\lambda| \geq L} \frac{1}{(n-\lambda)^{2}} \ll \lambda^{\eta} \int_{|x-\lambda| \geq L / 2} \frac{1}{(x-\lambda)^{2}} d x \ll \frac{\lambda^{\eta}}{L} .
\end{aligned}
$$

Thus, combining (3.2) with part (3) of Lemma 3.1, the bound (3.1) follows for the density one subset $\Lambda^{\prime}$ established in part (2) of Lemma 3.1.

We use the truncated Green's functions (2.5) to approximate the $L^{2}$-mass of $g_{\lambda}$ restricted to the ball $B_{x}(r)$.

Lemma 3.3. There exists a density one subset $\Lambda^{\prime} \subseteq \Lambda$, such that for every $\eta>0$, $0<\delta<1$, we have

$$
\left.\sup _{\substack{x \in \mathbb{T}^{2} \\ r \in \mathbb{R}}}\left|\int_{B_{x}(r)}\right| g_{\lambda}(y)\right|^{2} d y-\int_{B_{x}(r)}\left|g_{\lambda, L}(y)\right|^{2} d y \mid=O_{\eta}\left(\frac{\lambda^{\eta}}{L^{1 / 2}}\right)
$$

as $\lambda \rightarrow \infty$ along $\lambda \in \Lambda^{\prime}$, where $L=\lambda^{\delta}$.

Proof. Let $x \in \mathbb{T}^{2}, r \in \mathbb{R}$. Let $\mathbf{1}_{B_{x}(r)}$ be the indicator function of $B_{x}(r)$. Then by the Cauchy-Schwarz inequality and by (3.1),

$$
\begin{aligned}
& \left.\left|\int_{B_{x}(r)}\right| g_{\lambda}(y)\right|^{2} d y-\int_{B_{x}(r)}\left|g_{\lambda, L}(y)\right|^{2} d y \mid \\
& =\left|\left\langle\mathbf{1}_{B_{x}(r)} g_{\lambda}, g_{\lambda}\right\rangle-\left\langle\mathbf{1}_{B_{x}(r)} g_{\lambda, L}, g_{\lambda, L}\right\rangle\right| \\
& \leq\left|\left\langle\mathbf{1}_{B_{x}(r)}\left(g_{\lambda}-g_{\lambda, L}\right), g_{\lambda}\right\rangle\right|+\left|\left\langle\mathbf{1}_{B_{x}(r)} g_{\lambda, L}, g_{\lambda}-g_{\lambda, L}\right\rangle\right| \\
& \leq\left\|\mathbf{1}_{B_{x}(r)}\left(g_{\lambda}-g_{\lambda, L}\right)\right\|_{2}+\left\|\mathbf{1}_{B_{x}(r)} g_{\lambda, L}\right\|_{2}\left\|g_{\lambda}-g_{\lambda, L}\right\|_{2} \\
& \leq 2\left\|g_{\lambda}-g_{\lambda, L}\right\|_{2} \ll_{\eta} \frac{\lambda^{\eta}}{L^{1 / 2}},
\end{aligned}
$$


for $\lambda \in \Lambda^{\prime}$, the density one subset established in part (2) of Lemma 3.1. The statement of the lemma follows.

In light of Lemma 3.3, Theorem 1.1 will immediately follow from the following main proposition for the truncated Green's functions $g_{\lambda, L}$, which will be proved in the following subsections.

Proposition 3.4. There exists a density one subset $\Lambda^{\prime} \subseteq \Lambda$, such that for every $\epsilon>0$

$$
\left.\sup _{\substack{x \in \mathbb{T}^{2} \\ r>\lambda^{-1 / 2+\epsilon}}}\left|\frac{1}{\pi r^{2}} \int_{B_{x}(r)}\right| g_{\lambda, L}(y)\right|^{2} d y-1 \mid \rightarrow 0
$$

as $\lambda \rightarrow \infty$ along $\Lambda^{\prime}$, where $L=\lambda^{\delta}, 0<\delta<\epsilon / 6$.

Proof of Theorem 1.1 assuming Proposition 3.4. Take $\Lambda^{\prime} \subseteq \Lambda$ to be the intersection of the density one subsets whose existence has been established in Lemma 3.3 and Proposition 3.4, By Lemma 3.3, for every $x \in \mathbb{T}^{2}, r>\lambda^{-1 / 2+\epsilon}$, we have

$$
\left.\left|\frac{1}{\pi r^{2}} \int_{B_{x}(r)}\right| g_{\lambda}(y)\right|^{2} d y-1|\leq| \frac{1}{\pi r^{2}} \int_{B_{x}(r)}\left|g_{\lambda, L}(y)\right|^{2} d y-1 \mid+O_{\eta}\left(\lambda^{\eta-\delta / 2}\right)
$$

as $\lambda \rightarrow \infty$ along $\Lambda^{\prime}$. Hence,

$$
\begin{aligned}
\left.\sup _{\substack{x \in \mathbb{T}^{2} \\
r>\lambda^{-1 / 2+\epsilon}}}\left|\frac{1}{\pi r^{2}} \int_{B_{x}(r)}\right| g_{\lambda}(y)\right|^{2} d y-1 \mid & \leq\left.\sup _{\substack{x \in \mathbb{T}^{2} \\
r>\lambda^{-1 / 2+\epsilon}}}\left|\frac{1}{\pi r^{2}} \int_{B_{x}(r)}\right| g_{\lambda, L}(y)\right|^{2} d y-1 \mid \\
& +O_{\eta}\left(\lambda^{\eta-\delta / 2}\right)
\end{aligned}
$$

as $\lambda \rightarrow \infty$ along $\Lambda^{\prime}$. Theorem 1.1 now follows from Proposition [3.4, choosing $0<$ $\delta<\epsilon / 6$ and $\eta<\delta / 2$.

3.2. An $L^{2}$-mass expansion. In the following subsections, we prove Proposition 3.4. Our starting point is the following expansion. 
Lemma 3.5. Let $0<\delta<1, L=\lambda^{\delta}$. We have

$$
\begin{aligned}
& \int_{B_{x}(r)}\left|g_{\lambda, L}(y)\right|^{2} d y-\pi r^{2} \\
& =\frac{2 \pi r^{2}}{\left\|G_{\lambda, L}\right\|^{2}} \sum_{|n-\lambda|<L} \frac{1}{(n-\lambda)^{2}} \sum_{\substack{\xi \neq \xi^{\prime} \\
|\xi|^{2}=\left|\xi^{\prime}\right|^{2}=n}} e^{i\left\langle x-x_{0}, \xi-\xi^{\prime}\right\rangle} \cdot \frac{J_{1}\left(r\left|\xi-\xi^{\prime}\right|\right)}{r\left|\xi-\xi^{\prime}\right|} \\
& +\frac{2 \pi r^{2}}{\left\|G_{\lambda, L}\right\|^{2}} \sum_{\zeta \neq 0} e^{i\left\langle x-x_{0}, \zeta\right\rangle} \cdot \frac{J_{1}(r|\zeta|)}{r|\zeta|} \sum_{\substack{\left.|| \xi\right|^{2}-\lambda|<L\\
||\xi-\zeta|^{2}-\left.\lambda|<L\\
| \xi\right|^{2} \neq|\xi-\zeta|^{2}}} \frac{1}{\left(|\xi|^{2}-\lambda\right)\left(|\xi-\zeta|^{2}-\lambda\right)},
\end{aligned}
$$

where $J_{1}$ is the Bessel function of the first kind of order 1.

Proof. We expand

$$
\begin{gathered}
\int_{B_{x}(r)}\left|g_{\lambda, L}(y)\right|^{2} d y=\frac{1}{\left\|G_{\lambda, L}\right\|^{2}} \int_{B_{x}(r)} \sum_{\substack{\left.|| \xi\right|^{2}-\lambda|<L\\
|\left|\xi^{\prime}\right|^{2}-\lambda \mid<L}} \frac{e^{i\left\langle y-x_{0}, \xi-\xi^{\prime}\right\rangle}}{\left(|\xi|^{2}-\lambda\right)\left(\left|\xi^{\prime}\right|^{2}-\lambda\right)} d y \\
=\pi r^{2}+\frac{1}{\left\|G_{\lambda, L}\right\|^{2}} \sum_{\substack{\left.|| \xi\right|^{2}-\lambda|<L\\
|\left|\xi^{\prime}\right|^{2}-\lambda \mid<L \\
\xi \neq \xi^{\prime}}} \frac{e^{i\left\langle-x_{0}, \xi-\xi^{\prime}\right\rangle}}{\left(|\xi|^{2}-\lambda\right)\left(\left|\xi^{\prime}\right|^{2}-\lambda\right)} \int_{B_{x}(r)} e^{i\left\langle y, \xi-\xi^{\prime}\right\rangle} d y .
\end{gathered}
$$

Note that by the change of variable $z=\frac{y-x}{r}$, we have

$$
\int_{B_{x}(r)} e^{i\left\langle y, \xi-\xi^{\prime}\right\rangle} d y=r^{2} e^{i\left\langle x, \xi-\xi^{\prime}\right\rangle} \int_{B_{0}(1)} e^{i\left\langle z, r\left(\xi-\xi^{\prime}\right)\right\rangle} d z=2 \pi r^{2} e^{i\left\langle x, \xi-\xi^{\prime}\right\rangle} \frac{J_{1}\left(r\left|\xi-\xi^{\prime}\right|\right)}{\left|\xi-\xi^{\prime}\right|}
$$

where $J_{1}$ is the Bessel function of the first kind of order 1. Thus

$$
\int_{B_{x}(r)}\left|g_{\lambda, L}(y)\right|^{2} d y=\pi r^{2}+\frac{2 \pi r^{2}}{\left\|G_{\lambda, L}\right\|^{2}} \sum_{\substack{\left.|| \xi\right|^{2}-\left.\lambda|<L\\| \xi^{\prime}\right|^{2}-\lambda \mid<L \\ \xi \neq \xi^{\prime}}} \frac{e^{i\left\langle x-x_{0}, \xi-\xi^{\prime}\right\rangle}}{\left(|\xi|^{2}-\lambda\right)\left(\left|\xi^{\prime}\right|^{2}-\lambda\right)} \cdot \frac{J_{1}\left(r\left|\xi-\xi^{\prime}\right|\right)}{r\left|\xi-\xi^{\prime}\right|} .
$$

and (3.3) follows from the change of variable $\zeta=\xi-\xi^{\prime}$.

3.3. The first term in (3.3). We would like to evaluate the first term on the right-hand-side of equation (3.3). For $\epsilon>0$, let 


$$
B R(\epsilon)=\left\{n \in \mathcal{N}_{2}: \min _{\substack{\xi \neq \xi^{\prime} \\|\xi|^{2}=\left|\xi^{\prime}\right|^{2}=n}}\left|\xi-\xi^{\prime}\right| \leq n^{1 / 2-\epsilon}\right\}
$$

Bourgain and Rudnick [3] proved that

$$
\#\{n \in B R(\epsilon): n \leq X\}=O\left(X^{1-\epsilon / 3}\right) ;
$$

this bound was recently improved by Granville and Wigman [7] to

$$
\#\{n \in B R(\epsilon): n \leq X\}=O\left(X^{1-\epsilon}(\log X)^{1 / 2}\right)
$$

by rather sophisticated methods, though the bound (3.4) is sufficient for our application. On recalling (2.1), it follows that $B R(\epsilon)$ is a density zero set in $\mathcal{N}_{2}$, i.e., on most circles with radius $\sqrt{n}$, there are no lattice points with distance smaller or equal to $n^{1 / 2-\epsilon}$. The next lemma shows that most elements of $\Lambda$ are far from the elements of $B R(\epsilon)$.

Lemma 3.6. Let $\epsilon>0,0<\delta<1$. We have

$$
\#\{\lambda \in \Lambda: \lambda \leq X, \exists n \in B R(\epsilon) \cdot|n-\lambda| \leq L\}=O\left(X^{1-\epsilon / 3+\delta}\right)
$$

where $L=\lambda^{\delta}$.

Proof. We have

$$
\#\{\lambda \in \Lambda: \lambda \leq X, \exists n \in B R(\epsilon) .|n-\lambda| \leq L\} \leq \sum_{\substack{\lambda \in \Lambda \\ \lambda \leq X}} \sum_{\substack{n \in B R(\epsilon) \\|n-\lambda| \leq L}} 1 \leq \sum_{\substack{n \leq 2 X \\ n \in B R(\epsilon)}} \sum_{\substack{\lambda \in \Lambda \\ n \in \lambda \mid \leq X^{\delta}}} 1
$$

where in the second inequality we have changed the order of summation and replaced $L$ with $X^{\delta}$. The bound (3.5) follows from the trivial bound

$$
\#\left\{\lambda \in \Lambda:|n-\lambda| \leq X^{\delta}\right\} \ll X^{\delta}
$$

and the bound (3.4).

Choosing $L=\lambda^{\delta}$ with $0<\delta=\delta(\epsilon)<\epsilon / 3$, we conclude that the set

$$
\Lambda^{\prime \prime}(\epsilon)=\{\lambda \in \Lambda:|n-\lambda| \leq L \Longrightarrow n \notin B R(\epsilon)\}
$$

is a density one subset in $\Lambda$. From now on, we restrict to $\lambda \in \Lambda_{0}:=\Lambda^{\prime \prime}(\epsilon / 2)$, so that for $0<\delta=\delta(\epsilon)<\epsilon / 6, \Lambda_{0}$ is a density one subset in $\Lambda$. We are now ready to evaluate the first term on the right-hand-side of equation (3.3). 
Lemma 3.7. Let $\epsilon>0,0<\delta<\epsilon / 6, L=\lambda^{\delta}$. We have

$$
\sup _{\substack{x \in \mathbb{T}^{2} \\ r>\lambda^{1 / 2+\epsilon}}}\left|\frac{1}{\left\|G_{\lambda, L}\right\|^{2}} \sum_{|n-\lambda|<L} \frac{1}{(n-\lambda)^{2}} \sum_{\substack{\xi \neq \xi^{\prime} \\|\xi|^{2}=\left|\xi^{\prime}\right|^{2}=n}} e^{i\left\langle x-x_{0}, \xi-\xi^{\prime}\right\rangle} \cdot \frac{J_{1}\left(r\left|\xi-\xi^{\prime}\right|\right)}{r\left|\xi-\xi^{\prime}\right|}\right|=O\left(\lambda^{-\epsilon / 2}\right)
$$

along $\lambda \in \Lambda_{0}$.

Proof. Since $\lambda \in \Lambda_{0}=\Lambda^{\prime \prime}(\epsilon / 2)$, the inner summation on the left-hand-side of (3.6) can be restricted to $\left|\xi-\xi^{\prime}\right|>n^{1 / 2-\epsilon / 2}$. Since $J_{1}(x) \ll x^{-1 / 2}$, it follows that

$$
\begin{aligned}
\sum_{\substack{\xi \neq \xi^{\prime} \\
|\xi|^{2}=\left|\xi^{\prime}\right|^{2}=n}} e^{i\left\langle x-x_{0}, \xi-\xi^{\prime}\right\rangle} \cdot \frac{J_{1}\left(r\left|\xi-\xi^{\prime}\right|\right)}{r\left|\xi-\xi^{\prime}\right|} & \ll \frac{1}{r^{3 / 2}} \sum_{\substack{\xi \neq \xi^{\prime} \\
|\xi|^{2}=\left|\xi^{\prime}\right|^{2}=n \\
\left|\xi-\xi^{\prime}\right|>n^{1 / 2-\epsilon / 2}}} \frac{1}{\left|\xi-\xi^{\prime}\right|^{3 / 2}} \\
& \ll \frac{1}{r^{3 / 2}} r_{2}(n)^{2} \frac{1}{n^{3 / 4-3 \epsilon / 4}}
\end{aligned}
$$

and therefore the expression inside the supremum in (3.6) is bounded above by

$$
\frac{1}{\left\|G_{\lambda, L}\right\|^{2}} \sum_{|n-\lambda|<L} \frac{1}{(n-\lambda)^{2}} \frac{r_{2}(n)^{2}}{r^{3 / 2} n^{3 / 4-3 \epsilon / 4}} \ll \frac{\lambda^{-3 / 4+\epsilon}}{r^{3 / 2}}
$$

where we have used the bound (2.2) and the fact that

$$
\left\|G_{\lambda, L}\right\|^{2}=\sum_{|n-\lambda|<L} \frac{r_{2}(n)}{(n-\lambda)^{2}} .
$$

The estimate (3.6) now follows, since we take the supremum over $r>\lambda^{-1 / 2+\epsilon}$.

3.4. The second term in (3.3). Fix $\lambda \in \Lambda_{0}$. Our next goal is to evaluate the second term on the right-hand-side of equation (3.3). Note that

$$
|| \xi-\left.\zeta\right|^{2}-\lambda|=||\xi|^{2}-\lambda-2\langle\xi, \zeta\rangle+|\zeta|^{2} \mid
$$

so that

$$
|\zeta|^{2} \leq|| \xi-\left.\zeta\right|^{2}-\lambda|+||\xi|^{2}-\lambda|+2|\langle\xi, \zeta\rangle \mid
$$

Thus, if $\left.|| \xi\right|^{2}-\lambda \mid<L$ and ||$\xi-\left.\zeta\right|^{2}-\lambda \mid<L$, then using Cauchy-Schwarz inequality, (3.7) implies

$$
|\zeta|^{2}<2 L+2|\langle\xi, \zeta\rangle| \leq 5|\zeta| \sqrt{\lambda}
$$


and therefore the outer summation of the second term in (3.3) can be restricted to $|\zeta| \leq 5 \sqrt{\lambda}$. We can also write

$$
|| \xi-\left.\zeta\right|^{2}-\lambda|=||\xi|^{2}-\lambda-\langle 2 \xi-\zeta, \zeta\rangle \mid
$$

so that

$$
|\langle 2 \xi-\zeta, \zeta\rangle| \leq|| \xi-\left.\zeta\right|^{2}-\lambda|+||\xi|^{2}-\lambda \mid
$$

and therefore the inner summation of the second term in (3.3) can be restricted to $\xi \in \mathbb{Z}^{2}$ which satisfy $\left.|| \xi\right|^{2}-\lambda \mid<L$ and

$$
0<|\langle 2 \xi-\zeta, \zeta\rangle|<2 L
$$

and in particular (since $|\xi|^{2} \geq \lambda / 2$ for $\lambda \geq 4$, assuming $\delta<\frac{1}{2}$ ),

$$
0<|\langle 2 \xi-\zeta, \zeta\rangle| \leq 3|\xi|^{2 \delta}
$$

For every $\zeta \neq 0$, define

$$
A_{\zeta, \delta}=\left\{\xi \in \mathbb{Z}^{2}: 0<|\langle 2 \xi-\zeta, \zeta\rangle| \leq 3|\xi|^{2 \delta}\right\}
$$

and

$$
B_{\zeta, \delta}=\left\{\tilde{\xi} \in \mathbb{Z}^{2}: 0<|\langle\tilde{\xi}, \zeta\rangle| \leq 3\left|\frac{\tilde{\xi}+\zeta}{2}\right|^{2 \delta}\right\} .
$$

Note that for any $\xi \in A_{\zeta, \delta}$, we have $\tilde{\xi}:=2 \xi-\zeta \in B_{\zeta, \delta}$, and $|\xi|^{2} \leq 2 X$ implies $|\tilde{\xi}+\zeta|^{2} \leq 8 X$. Hence, for any $X$ sufficiently large such that $|\zeta| \leq 5 \sqrt{X}$, we have

$$
\begin{aligned}
\#\left\{\xi \in A_{\zeta, \delta}:|\xi|^{2} \leq 2 X\right\} & \leq \#\left\{\tilde{\xi} \in B_{\zeta, \delta}:|\tilde{\xi}+\zeta|^{2} \leq 8 X\right\} \\
& \leq \#\left\{\tilde{\xi} \in B_{\zeta, \delta}:|\tilde{\xi}| \leq|\zeta|+3 X^{1 / 2}\right\} \\
& \leq \#\left\{\tilde{\xi} \in B_{\zeta, \delta}:|\tilde{\xi}| \leq 8 X^{1 / 2}\right\} .
\end{aligned}
$$

Next, we estimate the right-hand-side of (3.8).

Lemma 3.8. Let $0<\delta<1 / 2, \zeta \neq 0$. For $X$ sufficiently large such that $|\zeta| \leq 5 \sqrt{X}$, we have

$$
\#\left\{\tilde{\xi} \in B_{\zeta, \delta}:|\tilde{\xi}| \leq 8 X^{1 / 2}\right\} \ll \frac{X^{1 / 2+\delta}}{|\zeta|} .
$$

Proof. Denote $\zeta=(m, n)$, and let $d=\operatorname{gcd}(m, n)$. For any $l \in \mathbb{Z}$, there is a solution to the equation

$$
\langle\tilde{\xi}, \zeta\rangle=l
$$


if and only if $d \mid l$, and then if $\tilde{\xi}_{0}$ is a solution, the other solutions are given by

$$
\tilde{\xi}=\tilde{\xi}_{0}+k\left(-\frac{n}{d}, \frac{m}{d}\right)
$$

where $k \in \mathbb{Z}$. Thus, for $l$ satisfying $d \mid l$, the number of solutions $\tilde{\xi}$ to (3.9) such that $|\tilde{\xi}| \leq 8 X^{1 / 2}$ is bounded above (up to a constant factor) by

$$
\frac{X^{1 / 2}}{\left|\left(-\frac{n}{d}, \frac{m}{d}\right)\right|}=\frac{d X^{1 / 2}}{|\zeta|}
$$

We deduce that

$$
\#\left\{\tilde{\xi} \in B_{\zeta, \delta}:|\tilde{\xi}| \leq 8 X^{1 / 2}\right\} \ll \frac{d X^{1 / 2}}{|\zeta|} \sum_{\substack{1 \leq|l| \leq 21 X^{\delta} \\ d \mid l}} 1 \ll \frac{X^{1 / 2+\delta}}{|\zeta|}
$$

(note that it is crucial that $l \neq 0$ for the last inequality to hold).

Next, we show that for most of the elements of $\lambda \in \Lambda_{0}$, if $\left.|| \xi\right|^{2}-\lambda \mid \leq L$, then $\xi$ is not an element of $A_{\zeta, \delta}$.

Lemma 3.9. Let $0<\delta<1 / 2, \zeta \neq 0$. For $X$ sufficiently large such that $|\zeta| \leq 5 \sqrt{X}$, we have

$$
\#\left\{\lambda \in \Lambda_{0}: \lambda \leq X,\left.\exists \xi \in A_{\zeta, \delta} \cdot|| \xi\right|^{2}-\lambda \mid \leq L\right\} \ll \frac{X^{1 / 2+2 \delta}}{|\zeta|}
$$

where $L=\lambda^{\delta}$.

Proof. We have

$$
\begin{aligned}
\#\left\{\lambda \in \Lambda_{0}: \lambda \leq X,\left.\exists \xi \in A_{\zeta, \delta} \cdot|| \xi\right|^{2}-\lambda \mid \leq L\right\} & \leq \sum_{\substack{\lambda \in \Lambda_{0} \\
\lambda \leq X}} \sum_{\substack{\left.\xi \in A_{\zeta, \delta} \\
|| \xi\right|^{2}-\lambda \mid \leq L}} 1 \\
& \leq \sum_{\substack{\xi \in A_{\zeta, \delta} \\
|\xi|^{2} \leq 2 X}} \sum_{\substack{\lambda \in \Lambda_{0} \\
|\xi|^{2}-\lambda \mid \leq X^{\delta}}} 1
\end{aligned}
$$

where in the last inequality we changed the order of summation and replaced $L=\lambda^{\delta}$ with $X^{\delta}$. By the trivial bound

$$
\left\{\lambda \in \Lambda_{0}:\left.|| \xi\right|^{2}-\lambda \mid \leq X^{\delta}\right\} \ll X^{\delta}
$$


and using the bound (3.8) and Lemma 3.8, we get

$$
\begin{aligned}
\#\left\{\lambda \in \Lambda_{0}: \lambda \leq X, \exists \xi \in A_{\zeta, \delta} \cdot|\xi|^{2}-\lambda \mid \leq L\right\} & \ll X^{\delta} \cdot \#\left\{\xi \in A_{\zeta, \delta}:|\xi|^{2} \leq 2 X\right\} \\
& \leq X^{\delta} \cdot \#\left\{\tilde{\xi} \in B_{\zeta, \delta}:|\tilde{\xi}| \leq 8 X^{1 / 2}\right\} \\
& \ll \frac{X^{1 / 2+2 \delta}}{|\zeta|} .
\end{aligned}
$$

For $\lambda \in \Lambda_{0}$ and $\zeta \neq 0$, denote

$$
\chi_{\zeta}(\lambda)= \begin{cases}1 & \left.\exists \xi \in A_{\zeta, \delta} \cdot|| \xi\right|^{2}-\lambda \mid \leq L \\ 0 & \text { otherwise. }\end{cases}
$$

Lemma 3.10. Let $\epsilon>0,0<\delta<\epsilon / 6, \lambda \in \Lambda_{0}, L=\lambda^{\delta}$. We have

$$
\left.\sup _{\substack{x \in \mathbb{T}^{2} \\ r>\lambda^{-1 / 2+\epsilon}}}\left|\frac{1}{\pi r^{2}} \int_{B_{x}(r)}\right| g_{\lambda, L}(y)\right|^{2} d y-1 \mid \ll \sum_{0<|\zeta| \leq 5 \sqrt{\lambda}} \chi_{\zeta}(\lambda) \cdot \sup _{r>\lambda^{-1 / 2+\epsilon}} \frac{\left|J_{1}(r|\zeta|)\right|}{r|\zeta|}+\lambda^{-\epsilon / 2}
$$

Proof. By (3.3) and by the bound (3.6), we have

$$
\left.\left|\int_{B_{x}(r)}\right| g_{\lambda, L}(y)\right|^{2} d y-\pi r^{2} \mid
$$

$$
\ll \frac{r^{2}}{\left\|G_{\lambda, L}\right\|^{2}} \sum_{\zeta \neq 0} \chi_{\zeta}(\lambda) \cdot \frac{\left|J_{1}(r|\zeta|)\right|}{r|\zeta|} \sum_{\substack{\left.|| \xi\right|^{2}-\lambda|<L\\||\xi-\zeta|^{2}-\left.\lambda|<L\\| \xi\right|^{2} \neq|\xi-\zeta|^{2}}} \frac{1}{\left.|| \xi\right|^{2}-\lambda|||\xi-\zeta|^{2}-\lambda \mid}+r^{2} \lambda^{-\epsilon / 2} .
$$

By the Cauchy-Schwarz inequality,

$$
\sum_{\substack{\left.|| \xi\right|^{2}-\lambda|<L\\||\xi-\zeta|^{2}-\left.\lambda|<L\\| \xi\right|^{2} \neq|\xi-\zeta|^{2}}} \frac{1}{\left.|| \xi\right|^{2}-\lambda|||\xi-\zeta|^{2}-\lambda \mid} \leq\left\|G_{\lambda, L}\right\|^{2}
$$

the bound (3.10) now follows substituting (3.12) into (3.11).

3.5. Proof of Proposition 3.4. Proposition 3.4 will follow from the following estimate, which we are now ready to prove. 
Lemma 3.11. Let $\epsilon>0,0<\delta<\epsilon / 6$. We have

$$
\left.\sum_{\substack{\lambda \in \Lambda_{0} \\ \lambda \leq X}} \sup _{\substack{x \in \mathbb{T}^{2} \\ r>\lambda^{-1 / 2+\epsilon}}}\left|\frac{1}{\pi r^{2}} \int_{B_{x}(r)}\right| g_{\lambda, L}(y)\right|^{2} d y-1 \mid=O\left(X^{1-\epsilon / 2}\right),
$$

where $L=\lambda^{\delta}$.

Proof. By (3.10), we have

$$
\begin{aligned}
& \left.\sum_{\substack{\lambda \in \Lambda_{0} \\
\lambda \leq X}} \sup _{\substack{x \in \mathbb{T}^{2} \\
r>\lambda^{-1 / 2+\epsilon}}}\left|\frac{1}{\pi r^{2}} \int_{B_{x}(r)}\right| g_{\lambda, L}(y)\right|^{2} d y-1 \mid \\
& \ll \sum_{\substack{\lambda \in \Lambda_{0} \\
\lambda \leq X}} \sum_{0<|\zeta| \leq 5 \sqrt{\lambda}} \chi_{\zeta}(\lambda) \cdot \sup _{r>X^{-1 / 2+\epsilon}} \frac{\left|J_{1}(r|\zeta|)\right|}{r|\zeta|}+\sum_{\substack{\lambda \in \Lambda_{0} \\
\lambda \leq X}} \lambda^{-\epsilon / 2} \\
& \ll \sum_{0<|\zeta| \leq 5 \sqrt{X}} \#\left\{\lambda \in \Lambda_{0}: \lambda \leq X,\left.\exists \xi \in A_{\zeta} \cdot|| \xi\right|^{2}-\lambda \mid \leq L\right\} \cdot \sup _{r>X^{-1 / 2+\epsilon}} \frac{\left|J_{1}(r|\zeta|)\right|}{r|\zeta|} \\
& +X^{14)} \ll \sum_{\zeta \neq 0} \sup _{r>X^{-1 / 2+\epsilon}} \frac{\left|J_{1}(r|\zeta|)\right|}{r|\zeta|^{2}}+X^{1-\epsilon / 2}
\end{aligned}
$$

where the last estimate follows from Lemma 3.9. Since $J_{1}(x) \ll \min \left\{x, x^{-1 / 2}\right\}$, we have

$$
\sup _{r>X^{-1 / 2+\epsilon}} \frac{\left|J_{1}(r|\zeta|)\right|}{r|\zeta|^{2}} \ll \min \left\{\frac{1}{|\zeta|}, \frac{X^{3 / 4-\frac{3}{2} \epsilon}}{|\zeta|^{5 / 2}}\right\}
$$

Thus

$$
\sum_{\zeta \neq 0} \sup _{r>X^{-1 / 2+\epsilon}} \frac{\left|J_{1}(r|\zeta|)\right|}{r|\zeta|^{2}} \ll \sum_{0<|\zeta| \leq X^{1 / 2-\epsilon}} \frac{1}{|\zeta|}+X^{3 / 4-\frac{3}{2} \epsilon} \sum_{|\zeta|>X^{1 / 2-\epsilon}} \frac{1}{|\zeta|^{5 / 2}} .
$$

We have (e.g., by comparison to an integral, or by partial summation)

$$
\sum_{0<|\zeta| \leq X^{1 / 2-\epsilon}} \frac{1}{|\zeta|} \ll X^{1 / 2-\epsilon}
$$

and

$$
X^{3 / 4-\frac{3}{2} \epsilon} \sum_{|\zeta|>X^{1 / 2-\epsilon}} \frac{1}{|\zeta|^{5 / 2}} \ll X^{1 / 2-\epsilon}
$$


Substituting the bounds (3.16) and (3.17) into (3.15) and then substituting back into (3.14), we get

$$
\left.\sum_{\substack{\lambda \in \Lambda_{0} \\ \lambda \leq X}} \sup _{\substack{x \in \lambda^{2} \\ r \in \lambda^{-1 / \epsilon}}}\left|\frac{1}{\pi r^{2}} \int_{B_{x}(r)}\right| g_{\lambda, L}(y)\right|^{2} d y-1 \mid \ll X^{1+2 \delta-\epsilon}+X^{1-\epsilon / 2} \ll X^{1-\epsilon / 2} .
$$

Proof of Proposition 3.4. Let $0<\delta=\delta(\epsilon)<\epsilon / 6$, and let

$$
B_{1}^{\epsilon}=\left\{\lambda \in \Lambda_{0}:\left.\sup _{\substack{x \in \mathbb{T}^{2} \\ r>\lambda^{-1 / 2+\epsilon}}}\left|\frac{1}{\pi r^{2}} \int_{B_{x}(r)}\right| g_{\lambda, L}(y)\right|^{2} d y-1 \mid>\lambda^{-\epsilon / 4}\right\} .
$$

Then by the Markov inequality and by Lemma 3.11,

$$
\left.\#\left\{\lambda \in B_{1}^{\epsilon}: \lambda \leq X\right\} \ll X^{\epsilon / 4} \sum_{\substack{\lambda \in \Lambda_{0} \\ \lambda \leq X}} \sup _{\substack{x \in \lambda^{-1 / 2+\epsilon} \\ r\left(\mathbb{T}^{2}\right.}}\left|\frac{1}{\pi r^{2}} \int_{B_{x}(r)}\right| g_{\lambda, L}(y)\right|^{2} d y-1 \mid \ll X^{1-\epsilon / 4} .
$$

Thus

$$
\Lambda_{1}^{\epsilon}:=\Lambda_{0} \backslash B_{1}^{\epsilon}=\left\{\lambda \in \Lambda_{0}:\left.\sup _{\substack{x \in \mathbb{T}^{2} \\ r>\lambda^{-1 / 2+\epsilon}}}\left|\frac{1}{\pi r^{2}} \int_{B_{x}(r)}\right| g_{\lambda, L}(y)\right|^{2} d y-1 \mid<\lambda^{-\epsilon / 4}\right\}
$$

is a density one subset in $\Lambda$. By a standard diagonal argument, we obtain a set $\Lambda_{1}$ of density one in $\Lambda$ which does not depend on $\epsilon$, such that for every $\epsilon>0$

$$
\left.\sup _{\substack{x \in \mathbb{T}^{2} \\ r>\lambda^{-1 / 2+\epsilon}}}\left|\frac{1}{\pi r^{2}} \int_{B_{x}(r)}\right| g_{\lambda, L}(y)\right|^{2} d y-1 \mid \rightarrow 0
$$

along $\lambda \in \Lambda_{1}$, completing the proof of Proposition 3.4 .

\section{Proofs of Theorem 1.2 and Theorem $1.3(d=3)$}

4.1. Preliminary lemmas. Let $d=3$, and recall that $\mathcal{N}_{3}$ is the set of numbers expressible as a sum of three squares, and that the elements of $\Lambda$ (the set of new eigenvalues) interlace with the elements of $\mathcal{N}_{3}$. We recall the following results proved in [29], which are the three-dimensional analogues of Lemma 3.1] and Lemma [3.2.

Lemma 4.1 ([29, Lemma 3.1, Lemma 3.2]).

(1) For all $\lambda \in \Lambda$ and all $\eta>0$, we have $\left\|G_{\lambda}\right\|^{2} \gg_{\eta} \lambda^{1 / 2-\eta}$.

(2) Let $0<\delta<1$. For all $\lambda \in \Lambda$ and all $\eta>0$, we have $\left\|g_{\lambda}-g_{\lambda, L}\right\|^{2} \ll_{\eta} \lambda^{-\delta+\eta}$ $\left(L=\lambda^{\delta}\right)$.

(3) For all $\lambda \in \Lambda$ and all $\eta>0$, we have $\left\|G_{\lambda, L}\right\|^{2} \gg_{\eta} \lambda^{1 / 2-\eta}\left(L=\lambda^{\delta}\right)$. 
As a corollary of Lemma 4.1, we state the following lemma, which is the threedimensional analogue of Lemma 3.3 .

Lemma 4.2. Let $0<\delta<1$. For every $\lambda \in \Lambda$ and every $\eta>0$, we have

$$
\left.\sup _{\substack{x \in \mathbb{T}^{3} \\ r \in \mathbb{R}}}\left|\int_{B_{x}(r)}\right| g_{\lambda}(y)\right|^{2} d y-\int_{B_{x}(r)}\left|g_{\lambda, L}(y)\right|^{2} d y \mid=O_{\eta}\left(\lambda^{\eta-\delta / 2}\right),
$$

where $L=\lambda^{\delta}$.

Proof. Similar to the proof of Lemma 3.3 .

Taking Lemma 4.2 into account, Theorem 1.2 immediately follows from the following proposition.

Proposition 4.3. Let $\epsilon>0$. We have

$$
\left.\sup _{\substack{x \in \mathbb{T}^{2} \\ r>\lambda^{-1 / 12+\epsilon}}}\left|\frac{1}{\frac{4}{3} \pi r^{3}} \int_{B_{x}(r)}\right| g_{\lambda, L}(y)\right|^{2} d y-1 \mid \rightarrow 0
$$

as $\lambda \rightarrow \infty$ along $\Lambda$, where $L=\lambda^{\delta}, 0<\delta<\epsilon$.

Proof of Theorem 1.2 assuming Proposition 4.3. By Lemma 4.2, for every $x \in \mathbb{T}^{3}$, $r>\lambda^{-1 / 12+\epsilon}$, we have

$$
\left.\left|\frac{1}{\frac{4}{3} \pi r^{3}} \int_{B_{x}(r)}\right| g_{\lambda}(y)\right|^{2} d y-1|\leq| \frac{1}{\frac{4}{3} \pi r^{3}} \int_{B_{x}(r)}\left|g_{\lambda, L}(y)\right|^{2} d y-1 \mid+O_{\eta}\left(\lambda^{\eta-\delta / 2}\right) .
$$

Hence,

$$
\begin{aligned}
\left.\sup _{\substack{x \in \mathbb{T}^{2} \\
r>\lambda^{-1 / 12+\epsilon}}}\left|\frac{1}{\frac{4}{3} \pi r^{3}} \int_{B_{x}(r)}\right| g_{\lambda}(y)\right|^{2} d y-1 \mid & \leq\left.\sup _{\substack{x \in \mathbb{T}^{2} \\
r>\lambda^{-1 / 12+\epsilon}}}\left|\frac{1}{\frac{4}{3} \pi r^{3}} \int_{B_{x}(r)}\right| g_{\lambda, L}(y)\right|^{2} d y-1 \mid \\
& +O_{\eta}\left(\lambda^{\eta-\delta / 2}\right) .
\end{aligned}
$$

Theorem 1.2 now follows from Proposition 4.3, choosing $\delta<\epsilon$ and $\eta<\delta / 2$.

We will use the following approximation to the characteristic function of a ball of radius $r$ ("Beurling-Selberg polynomials") appearing in the work of Harman [11].

Lemma 4.4 ([11, Lemma 4]). Let T, $r>0$ such that $\operatorname{Tr}>1$. There exist trigonometric polynomials $a^{ \pm}$such that:

(1) $a^{-}(y) \leq \mathbf{1}_{B_{0}(r)}(y) \leq a^{+}(y)$.

(2) $\hat{a}^{ \pm}(\zeta)=0$ if $|\zeta| \geq T$.

(3) $\hat{a}^{ \pm}(0)=\operatorname{vol}\left(B_{0}(r)\right)+O_{d}\left(r^{d-1} / T\right)$.

(4) $\left|\hat{a}^{ \pm}(\zeta)\right| \ll_{d} r^{d}$. 
Given $x \in \mathbb{T}^{3}$, the polynomials $b_{x}^{ \pm}(y)=a^{ \pm}(y-x)$ satisfy

$$
b_{x}^{-}(y) \leq \mathbf{1}_{B_{x}(r)}(y) \leq b_{x}^{+}(y)
$$

and also satisfy properties (2)-(4) of Lemma 4.4. Proposition 4.3 will follow from the following proposition, which will be proved in the following subsections.

Proposition 4.5. Let $\epsilon>0$. We have

$$
\left.\sup _{\substack{x \in \mathbb{T}^{2} \\ r>\lambda^{-1 / 12+\epsilon}}}\left|\frac{1}{\frac{4}{3} \pi r^{3}} \int_{\mathbb{T}^{3}} b_{x}^{ \pm}(y)\right| g_{\lambda, L}(y)\right|^{2} d y-1 \mid \rightarrow 0
$$

as $\lambda \rightarrow \infty$ along $\Lambda$, where $L=\lambda^{\delta}, 0<\delta<\epsilon$.

Proof of Proposition 4.3 assuming Proposition 4.5. For every $x \in \mathbb{T}^{3}, r>\lambda^{-1 / 12+\epsilon}$, the bounds (4.1) imply

$$
\begin{aligned}
\frac{1}{\frac{4}{3} \pi r^{3}} \int_{\mathbb{T}^{3}} b_{x}^{-}(y)\left|g_{\lambda, L}(y)\right|^{2} d y & \leq \frac{1}{\frac{4}{3} \pi r^{3}} \int_{B_{x}(r)}\left|g_{\lambda, L}(y)\right|^{2} d y \\
& \leq \frac{1}{\frac{4}{3} \pi r^{3}} \int_{\mathbb{T}^{3}} b_{x}^{+}(y)\left|g_{\lambda, L}(y)\right|^{2} d y
\end{aligned}
$$

Proposition 4.3 now clearly follows from Proposition 4.5.

4.2. An $L^{2}$-mass expansion. Our starting point towards proving Proposition 4.5, as well as proving Theorem [1.3, is the following expansion.

Lemma 4.6. Let $T, r>0$ such that $\operatorname{Tr}>1$, and let $0<\delta<1, L=\lambda^{\delta}$. We have

$$
\int_{\mathbb{T}^{3}} b_{x}^{ \pm}(y)\left|g_{\lambda, L}(y)\right|^{2} d y-\frac{4}{3} \pi r^{3}
$$

$$
=\frac{1}{\left\|G_{\lambda, L}\right\|^{2}} \sum_{0<|\zeta|<T} \hat{b}_{x}^{ \pm}(\zeta) e^{-i\left\langle x_{0}, \zeta\right\rangle} \sum_{\substack{\left.|| \xi\right|^{2}-\lambda|<L\\||\xi-\zeta|^{2}-\lambda \mid<L}} \frac{1}{\left(|\xi|^{2}-\lambda\right)\left(|\xi-\zeta|^{2}-\lambda\right)}+O\left(r^{2} / T\right) .
$$

Proof. We expand

$$
\int_{\mathbb{T}^{3}} b_{x}^{ \pm}(y)\left|g_{\lambda, L}(y)\right|^{2} d y=\frac{1}{\left\|G_{\lambda, L}\right\|^{2}} \sum_{\substack{\left.|| \xi\right|^{2}-\lambda|<L\\|\left|\xi^{\prime}\right|^{2}-\lambda \mid<L}} \frac{e^{-i\left\langle x_{0}, \xi-\xi^{\prime}\right\rangle}}{\left(|\xi|^{2}-\lambda\right)\left(\left|\xi^{\prime}\right|^{2}-\lambda\right)} \hat{b}_{x}^{ \pm}\left(\xi-\xi^{\prime}\right)
$$

and writing $\zeta=\xi-\xi^{\prime}$, the lemma follows from properties (2) and (3) of Lemma 4.4.

For each $\zeta \neq 0$, denote $|\zeta|^{2}=n_{\zeta}=4^{a_{\zeta}} n_{1}^{\zeta}$ with $4 \nmid n_{1}^{\zeta}$. 
Definition 4.7. Define

$$
\mathcal{N}_{0}^{\zeta}=\left\{n \in \mathcal{N}_{3}: n=4^{a} n_{1}, 4 \nmid n_{1} \Rightarrow a>a_{\zeta}\right\}
$$

and

$$
\mathcal{N}_{1}^{\zeta}=\left\{n \in \mathcal{N}_{3}: n=4^{a} n_{1}, 4 \nmid n_{1} \Rightarrow a \leq a_{\zeta}\right\} .
$$

Denote by $n_{\lambda}$ the element of $\mathcal{N}_{3}$ which is closest to $\lambda \in \Lambda$ (if there are two elements with the same distance, we take the $n_{\lambda}$ to be the smallest among them). We recall the following results, proved in [29].

Lemma 4.8 ([29, Lemma 3.6, Corollary 3.7, Lemma 3.8]).

(1) For every $\xi \in \mathbb{Z}^{3}$, if $|\xi|^{2}=|\xi-\zeta|^{2}$, then $|\xi|^{2} \in \mathcal{N}_{1}^{\zeta}$.

(2) For every $\xi \in \mathbb{Z}^{3}$, if $|\xi|^{2} \in \mathcal{N}_{0}^{\zeta}$, then $\left.|| \xi\right|^{2}-|\xi-\zeta|^{2} \mid \geq 1$.

(3) If $n_{\lambda} \in \mathcal{N}_{0}^{\zeta}$, then $\left.|| \xi\right|^{2}-\lambda|<1 / 2 \Rightarrow||\xi-\zeta|^{2}-\lambda \mid>1 / 2$, for every $\xi \in \mathbb{Z}^{3}$.

To evaluate the right-hand-side of (4.2), we consider separately the summation over $0<|\zeta|<T$ such that $n_{\lambda} \in \mathcal{N}_{0}^{\zeta}$, and over $0<|\zeta|<T$ such that $n_{\lambda} \in \mathcal{N}_{1}^{\zeta}$. Our main tool for evaluating the sums in both cases, will be the following estimate for the number of lattice points inside spherical strips, which is a uniform version of [29, Lemma A.1].

Lemma 4.9. Let $C_{1}, C_{2}, C_{3}>0,0<\delta<1, L=\lambda^{\delta}, 0<|\zeta| \leq C_{1} \sqrt{\lambda}$. For every $n \in \mathcal{N}_{3}$ satisfying $|n-\lambda|<C_{2} L$ and every $\eta>0$, we have

$$
\#\left\{\xi \in \mathbb{Z}^{3}:|\xi|^{2}=n,|\langle 2 \xi-\zeta, \zeta\rangle|<C_{3} L\right\} \ll_{C_{1}, C_{2}, C_{3}, \eta} L \lambda^{\eta} .
$$

Proof. Denote $\zeta=\left(\zeta_{1}, \zeta_{2}, \zeta_{3}\right)$, and assume without loss of generality that $\zeta_{3} \neq 0$. Let $n \in \mathcal{N}_{3}$ so that $|n-\lambda|<C_{2} L$, so we are looking for $\xi=(x, y, z)$ such $|\xi|^{2}=n$, and such that $\langle 2 \xi-\zeta, \zeta\rangle=m$, where $|m|<C_{3} L$. In this notation, we have

$$
x^{2}+y^{2}+z^{2}=n
$$

and

$$
2 x \zeta_{1}+2 y \zeta_{2}+2 z \zeta_{3}=m+|\zeta|^{2} .
$$

Since $\zeta_{3} \neq 0$, we can write

$$
z=\frac{m+|\zeta|^{2}-2 \zeta_{1} x-2 \zeta_{2} y}{2 \zeta_{3}},
$$

and substituting (4.4) into (4.3), we get

$$
a x^{2}+2 b x y+c y^{2}+2 d x+2 e y+f=0
$$


where

$$
\begin{aligned}
a & =4 \zeta_{1}^{2}+4 \zeta_{3}^{2} \\
b & =4 \zeta_{1} \zeta_{2} \\
c & =4 \zeta_{2}^{2}+4 \zeta_{3}^{2} \\
d & =-2 \zeta_{1}\left(m+|\zeta|^{2}\right) \\
e & =-2 \zeta_{2}\left(m+|\zeta|^{2}\right) \\
f & =-4 \zeta_{3}^{2} n+\left(m+|\zeta|^{2}\right)^{2} .
\end{aligned}
$$

Note that $c>0, a c-b^{2}=16 \zeta_{3}^{2}|\zeta|^{2}>0$, and denote $a c-b^{2}=t^{2} D$, where $D>0$ is squarefree. By a simple sequence of changes of variables (see the proof of [29, Lemma A.1]), the number of integer solutions to equation (4.5) is bounded above by the number of integer solutions to the equation

$$
x^{2}+D y^{2}=k,
$$

where

$$
k=\left(a c-b^{2}\right)\left(-c f+e^{2}\right)+(c d-b e)^{2} .
$$

In the proof of [29, Lemma A.1]), we obtained that the number of integer solutions to equation (4.6) is bounded above by $6 \tau(k)$, where $\tau(k) \ll_{\eta} k^{\eta}$ is the number of divisors of $k$. Since $|\zeta| \leq C_{1} \sqrt{\lambda}$ and $|n-\lambda|<C_{2} L$, we deduce that the number of solutions is $\ll_{C_{1}, C_{2}, C_{3}, \eta} \lambda^{\eta}$ for every fixed $m$ such that $|m|<C_{3} L$. Taking into account the various choices for $m$ we get that the number of solutions is $\ll_{C_{1}, C_{2}, C_{3}, \eta} L \lambda^{\eta}$.

4.3. The case $n_{\lambda} \in \mathcal{N}_{0}^{\zeta}$. In light of Lemma 4.8, for every $0<|\zeta|<T$ such that $n_{\lambda} \in \mathcal{N}_{0}^{\zeta}$, we can split the inner summation on the right-hand-side of (4.2) into three sums over the following ranges:

(1) $\frac{1}{2} \leq\left.|| \xi\right|^{2}-\lambda\left|<L, \frac{1}{2} \leq\right||\xi-\zeta|^{2}-\lambda \mid \leq L$ (Denote this sum by $\Sigma^{1}$ ).

(2) $\left.|| \xi\right|^{2}-\lambda\left|<\frac{1}{2}, \frac{1}{2} \leq\right||\xi-\zeta|^{2}-\lambda \mid \leq L$ (Denote this sum by $\Sigma^{2}$ ).

(3) $\frac{1}{2} \leq\left.|| \xi\right|^{2}-\lambda|<L,||\xi-\zeta|^{2}-\lambda \mid<\frac{1}{2}$ (Denote this sum by $\sum^{3}$ ).

We will use Lemma 4.9 to estimate $\sum^{1}, \Sigma^{2}$ and $\sum^{3}$, which will give the required bound in the case $n_{\lambda} \in \mathcal{N}_{0}^{\zeta}$.

Lemma 4.10. Let $0<\delta<1, L=\lambda^{\delta}$. For every fixed $0<|\zeta|<T$ such that $n_{\lambda} \in \mathcal{N}_{0}^{\zeta}$ and every $\eta>0$, we have

$$
\sum_{\substack{\left.|| \xi\right|^{2}-\lambda|<L\\||\xi-\zeta|^{2}-\lambda \mid<L}} \frac{1}{\left(|\xi|^{2}-\lambda\right)\left(|\xi-\zeta|^{2}-\lambda\right)} \ll_{\eta} \lambda^{2 \delta+\eta}+\left\|G_{\lambda, L}\right\| \lambda^{\delta / 2+\eta / 2} .
$$


Proof. Recall that if $\left.|| \xi\right|^{2}-\lambda \mid<L$ and ||$\xi-\left.\zeta\right|^{2}-\lambda \mid<L$, then

$$
|\langle 2 \xi-\zeta, \zeta\rangle|=\left.|| \xi\right|^{2}-|\xi-\zeta|^{2}|\leq||\xi|^{2}-\lambda|+||\xi-\zeta|^{2}-\lambda \mid<2 L
$$

By Lemma 4.9, we have

$$
\begin{aligned}
\sum^{1} \frac{1}{\left(|\xi|^{2}-\lambda\right)\left(|\xi-\zeta|^{2}-\lambda\right)} & \ll \sum^{1} 1 \\
& \ll \sum_{|n-\lambda|<L} \#\left\{\xi \in \mathbb{Z}^{3}:|\xi|^{2}=n,|\langle 2 \xi-\zeta, \zeta\rangle|<2 L\right\} \\
& \ll_{\eta} L \lambda^{\eta} \sum_{|n-\lambda|<L} 1 \ll L^{2} \lambda^{\eta}=\lambda^{2 \delta+\eta} .
\end{aligned}
$$

Next, using the Cauchy-Schwarz inequality and Lemma 4.9 again, we have

$$
\begin{aligned}
& \sum^{2} \frac{1}{\left(|\xi|^{2}-\lambda\right)\left(|\xi-\zeta|^{2}-\lambda\right)} \ll \sum^{2} \frac{1}{\left.|| \xi\right|^{2}-\lambda \mid} \ll\left\|G_{\lambda, L}\right\|\left(\sum^{2} 1\right)^{1 / 2} \\
& \ll\left\|G_{\lambda, L}\right\|\left(\#\left\{\xi \in \mathbb{Z}^{3}:|\xi|^{2}=n_{\lambda},|\langle 2 \xi-\zeta, \zeta\rangle|<2 L\right\}\right)^{1 / 2} \\
& \ll_{\eta}\left\|G_{\lambda, L}\right\| L^{1 / 2} \lambda^{\eta / 2}=\left\|G_{\lambda, L}\right\| \lambda^{\delta / 2+\eta / 2} .
\end{aligned}
$$

The sum $\sum^{3}$ is treated like $\sum^{2}$ and is bounded by the same quantity.

We deduce a bound for the sum on the right-hand-side of (4.2) restricted to $0<$ $|\zeta|<T$ such that $n_{\lambda} \in \mathcal{N}_{0}^{\zeta}$.

Corollary 4.11. Let $T, r>0$ such that $T r>1$, and let $0<\delta<1, L=\lambda^{\delta}$. For every $\eta>0$, we have

$$
\begin{aligned}
& \sum_{\substack{0<|\zeta|<T \\
n_{\lambda} \in \mathcal{N}_{0}^{\zeta}}} \hat{b}_{x}^{ \pm}(\zeta) e^{-i\left\langle x_{0}, \zeta\right\rangle} \sum_{\substack{\left.|| \xi\right|^{2}-\lambda|<L\\
||\xi-\zeta|^{2}-\lambda \mid<L}} \frac{1}{\left(|\xi|^{2}-\lambda\right)\left(|\xi-\zeta|^{2}-\lambda\right)} \\
& \ll_{\eta} r^{3} T^{3}\left(\lambda^{2 \delta+\eta}+\left\|G_{\lambda, L}\right\| \lambda^{\delta / 2+\eta / 2}\right) .
\end{aligned}
$$

Proof. This follows immediately from Lemma 4.10 and property (4) of Lemma 4.4.

\subsection{The case $n_{\lambda} \in \mathcal{N}_{1}^{\zeta}$.}


Lemma 4.12. Let $0<\delta<1, L=\lambda^{\delta}$. For every fixed $0<|\zeta|<T$ such that $n_{\lambda} \in \mathcal{N}_{1}^{\zeta}$ and every $\eta>0$, we have

$$
\sum_{\substack{\left.|| \xi\right|^{2}-\lambda|<L\\||\xi-\zeta|^{2}-\lambda \mid<L}} \frac{1}{\left(|\xi|^{2}-\lambda\right)\left(|\xi-\zeta|^{2}-\lambda\right)} \ll_{\eta} \lambda^{2 \delta+\eta}+\left\|G_{\lambda, L}\right\|^{2} T \lambda^{\delta-\frac{1}{2}+2 \eta} .
$$

Proof. By the Cauchy-Schwarz inequality,

$$
\begin{aligned}
& \sum_{\substack{\left.|| \xi\right|^{2}-\lambda|<L\\
||\xi-\zeta|^{2}-\lambda \mid<L}} \frac{1}{\left(|\xi|^{2}-\lambda\right)\left(|\xi-\zeta|^{2}-\lambda\right)} \\
\ll & \left(\sum_{\substack{\left.|| \xi\right|^{2}-\lambda|<L\\
|\langle 2 \xi-\zeta, \zeta\rangle \mid<2 L}} \frac{1}{\left(|\xi|^{2}-\lambda\right)^{2}}\right)^{1 / 2}\left(\sum_{\substack{|| \xi-\left.\zeta\right|^{2}-\lambda|<L\\
|\langle 2 \xi-\zeta, \zeta\rangle \mid<2 L}} \frac{1}{\left(|\xi-\zeta|^{2}-\lambda\right)^{2}}\right)^{1 / 2} .
\end{aligned}
$$

Consider the first summation on the right-hand-side of (4.8)

$$
\sum_{\substack{\left.|| \xi\right|^{2}-\lambda|<L\\|\langle 2 \xi-\zeta, \zeta\rangle \mid<2 L}} \frac{1}{\left(|\xi|^{2}-\lambda\right)^{2}}
$$

and split it into two sums $\sum^{4}+\sum^{5}$, where in $\sum^{4}$ the summation is over $\xi$ such that $|\xi|^{2} \neq n_{\lambda},\left.|| \xi\right|^{2}-\lambda \mid<L$ and $|\langle 2 \xi-\zeta, \zeta\rangle|<2 L$, and in $\sum^{5}$ the summation is over $\xi$ such that $|\xi|^{2}=n_{\lambda}$ and $|\langle 2 \xi-\zeta, \zeta\rangle|<2 L$.

If $|\xi|^{2} \neq n_{\lambda}$, then $\left.|| \xi\right|^{2}-\lambda \mid \geq \frac{1}{2}$, and therefore Lemma 4.9 yields

$$
\begin{aligned}
\sum^{4} \frac{1}{\left(|\xi|^{2}-\lambda\right)^{2}} & \ll \sum_{\substack{\left.|| \xi\right|^{2}-\lambda|<L\\
|\langle 2 \xi-\zeta, \zeta\rangle \mid<2 L}} 1 \ll \sum_{|n-\lambda|<L} \#\left\{\xi \in \mathbb{Z}^{3}:|\xi|^{2}=n,|\langle 2 \xi-\zeta, \zeta\rangle|<2 L\right\} \\
& \ll_{\eta} \sum_{|n-\lambda|<L} L \lambda^{\eta} \ll L^{2} \lambda^{\eta}=\lambda^{2 \delta+\eta} .
\end{aligned}
$$

To bound $\sum^{5}$, note that by Lemma 4.9,

$$
\begin{aligned}
\sum^{5} \frac{1}{\left(|\xi|^{2}-\lambda\right)^{2}} & =\frac{1}{\left(n_{\lambda}-\lambda\right)^{2}} \#\left\{\xi \in \mathbb{Z}^{3}:|\xi|^{2}=n_{\lambda},|\langle 2 \xi-\zeta, \zeta\rangle|<2 L\right\} \\
& \ll_{\eta} \frac{L \lambda^{\eta}}{\left(n_{\lambda}-\lambda\right)^{2}}
\end{aligned}
$$


and therefore

$$
\frac{\sum^{5} \frac{1}{\left(|\xi|^{2}-\lambda\right)^{2}}}{\left\|G_{\lambda, L}\right\|^{2}} \ll_{\eta} \frac{\frac{L \lambda^{\eta}}{\left(n_{\lambda}-\lambda\right)^{2}}}{\left\|G_{\lambda, L}\right\|^{2}} \ll \frac{\frac{L \lambda^{\eta}}{\left(n_{\lambda}-\lambda\right)^{2}}}{\frac{r_{3}\left(n_{\lambda}\right)}{\left(n_{\lambda}-\lambda\right)^{2}}}=\frac{L \lambda^{\eta}}{r_{3}\left(n_{\lambda}\right)} .
$$

But since $n_{\lambda} \in \mathcal{N}_{1}^{\zeta}$, if we write $n_{\lambda}=4^{a} n_{1}$ with $4 \nmid n_{1}$, then $n_{\lambda} \leq 4^{a_{\zeta}} n_{1}$. Moreover, $2^{a_{\zeta}} \leq T$, and therefore, by (2.3),

$$
\frac{L \lambda^{\eta}}{r_{3}\left(n_{\lambda}\right)}=\frac{\lambda^{\delta+\eta}}{r_{3}\left(n_{1}\right)} \ll_{\eta} \frac{\lambda^{\delta+\eta}}{n_{1}^{1 / 2-\eta}} \ll \frac{2^{a_{\zeta}} \lambda^{\delta+\eta}}{n_{\lambda}^{1 / 2-\eta}} \ll T \lambda^{\delta-\frac{1}{2}+2 \eta},
$$

SO

$$
\sum^{5} \frac{1}{\left(|\xi|^{2}-\lambda\right)^{2}} \ll_{\eta}\left\|G_{\lambda, L}\right\|^{2} T \lambda^{\delta-\frac{1}{2}+2 \eta} .
$$

As for the second summation on the right-hand-side of (4.8), note that

$$
\sum_{\substack{|| \xi-\left.\zeta\right|^{2}-\lambda|<L\\|\langle 2 \xi-\zeta, \zeta\rangle \mid<2 L}} \frac{1}{\left(|\xi-\zeta|^{2}-\lambda\right)^{2}}=\sum_{\substack{\left.|| \tilde{\xi}\right|^{2}-\lambda|<L\\|\langle 2 \tilde{\xi}+\zeta, \zeta\rangle \mid<2 L}} \frac{1}{\left(|\tilde{\xi}|^{2}-\lambda\right)^{2}}
$$

and this summation can be bounded in the same way. Therefore

$$
\sum_{\substack{\left.|| \xi\right|^{2}-\lambda|<L\\||\xi-\zeta|^{2}-\lambda \mid<L}} \frac{1}{\left(|\xi|^{2}-\lambda\right)\left(|\xi-\zeta|^{2}-\lambda\right)} \ll_{\eta} \lambda^{2 \delta+\eta}+\left\|G_{\lambda, L}\right\|^{2} T \lambda^{\delta-\frac{1}{2}+2 \eta} .
$$

We deduce a bound for the sum on the right-hand-side of (4.2), now restricted to $0<|\zeta|<T$ such that $n_{\lambda} \in \mathcal{N}_{1}^{\zeta}$.

Corollary 4.13. Let $T, r>0$ such that $\operatorname{Tr}>1$, and let $0<\delta<1, L=\lambda^{\delta}$. For every $\eta>0$, we have

$$
\begin{aligned}
& \sum_{\substack{0<|\zeta|<T \\
n_{\lambda} \in \mathcal{N}_{1}^{\zeta}}} \hat{b}_{x}^{ \pm}(\zeta) e^{-i\left\langle x_{0}, \zeta\right\rangle} \sum_{\substack{\left.|| \xi\right|^{2}-\lambda|<L\\
||\xi-\zeta|^{2}-\lambda \mid<L}} \frac{1}{\left(|\xi|^{2}-\lambda\right)\left(|\xi-\zeta|^{2}-\lambda\right)} \\
& \ll_{\eta} r^{3} T^{3}\left(\lambda^{2 \delta+\eta}+\left\|G_{\lambda, L}\right\|^{2} T \lambda^{\delta-\frac{1}{2}+2 \eta}\right) .
\end{aligned}
$$

Proof. This follows immediately from Lemma 4.12 and property (4) of Lemma 4.4. 


\subsection{Proof of Proposition 4.5. We are now able to prove Proposition 4.5.}

Proof of Proposition 4.5. Substituting (4.7) and (4.13) into the right-hand-side of (4.2) and using the third part of Lemma 4.1, we conclude that for $r, T>0$ such that $\operatorname{Tr}>1$, we have

$$
\begin{aligned}
\int_{\mathbb{T}^{3}} b_{x}^{ \pm}(y)\left|g_{\lambda, L}(y)\right|^{2} d y-\frac{4}{3} \pi r^{3} & \ll \eta r^{3} T^{3}\left(\frac{\lambda^{2 \delta+\eta}}{\left\|G_{\lambda, L}\right\|^{2}}+\frac{\lambda^{\frac{\delta}{2}+\frac{\eta}{2}}}{\left\|G_{\lambda, L}\right\|}+T \lambda^{\delta-\frac{1}{2}+2 \eta}\right)+\frac{r^{2}}{T} \\
& \ll r^{3} T^{4} \lambda^{\delta-\frac{1}{2}+2 \eta}+r^{3} T^{3}\left(\lambda^{2 \delta-\frac{1}{2}+2 \eta}+\lambda^{\frac{\delta}{2}-\frac{1}{4}+\eta}\right)+\frac{r^{2}}{T} .
\end{aligned}
$$

Choose $T=\lambda^{1 / 12-\frac{\epsilon}{2}}$. If $r>\lambda^{-1 / 12+\epsilon}$, then $\operatorname{Tr}>1$, and hence if we take $\eta=\epsilon$,

$$
\int_{\mathbb{T}^{3}} b_{x}^{ \pm}(y)\left|g_{\lambda, L}(y)\right|^{2} d y-\frac{4}{3} \pi r^{3} \ll r^{3}\left(\lambda^{\frac{\delta}{2}-\frac{\epsilon}{2}}+\frac{1}{r \lambda^{\frac{1}{12}-\frac{\epsilon}{2}}}\right) \ll r^{3}\left(\lambda^{\frac{\delta}{2}-\frac{\epsilon}{2}}+\lambda^{-\frac{\epsilon}{2}}\right)
$$

and the statement of the proposition follows by choosing $0<\delta<\epsilon$.

4.6. Proof of Theorem 1.3. In this subsection, we prove Theorem 1.3. Denote

$$
\widetilde{\mathcal{N}}_{1}=\left\{n \in \mathcal{N}_{3}: n=4^{a} n_{1}, 4 \nmid n_{1} \Rightarrow n_{1}>n / \log ^{2} n\right\} .
$$

Lemma 4.14. The set $\widetilde{\mathcal{N}_{1}}$ is a density one subset in $\mathcal{N}_{3}$.

Proof. Consider the complement set

$$
\widetilde{\mathcal{N}}_{0}=\mathcal{N}_{3} \backslash \widetilde{\mathcal{N}}_{1}=\left\{n \in \mathcal{N}_{3}: n=4^{a} n_{1}, 4 \nmid n_{1} \Rightarrow n_{1} \leq n / \log ^{2} n\right\} .
$$

Let $X>0$, and assume that $n \in \widetilde{\mathcal{N}}_{0}$ and $n \leq X$. Then $a \leq \log _{4} X$, and $n_{1} \leq$ $X / \log ^{2} X$. Since $n$ is uniquely determined by $a$ and $n_{1}$, we conclude that

$$
\#\left\{n \in \widetilde{\mathcal{N}}_{0}: n \leq X\right\} \ll X / \log X
$$

so $\widetilde{\mathcal{N}}_{0}$ is a density zero set in $\mathcal{N}_{3}$, and therefore $\widetilde{\mathcal{N}}_{1}$ is a density one subset in $\mathcal{N}_{3}$.

Corollary 4.15. The set

$$
\Lambda^{\prime}=\left\{\lambda \in \Lambda: n_{\lambda} \in \widetilde{\mathcal{N}}_{1}\right\}
$$

is a density one subset in $\Lambda$.

Proof. This is an immediate corollary of Lemma 4.14, since the elements of $\Lambda$ interlace with the elements of $\mathcal{N}_{3}$.

Lemma 4.16. Let $\epsilon>0$. We have 


$$
\left.\sup _{\substack{x \in \mathbb{T}^{2} \\ r>\lambda^{-1 / 6+\epsilon}}}\left|\frac{1}{\frac{4}{3} \pi r^{3}} \int_{\mathbb{T}^{3}} b_{x}^{ \pm}(y)\right| g_{\lambda, L}(y)\right|^{2} d y-1 \mid \rightarrow 0
$$

as $\lambda \rightarrow \infty$ along $\Lambda^{\prime}$, where $L=\lambda^{\delta}, 0<\delta<\epsilon / 16$.

Proof. Recall that by Lemma 4.6, for $T, r>0$ such that $\operatorname{Tr}>1$, we have

$$
\begin{aligned}
& \int_{\mathbb{T}^{3}} b_{x}^{ \pm}(y)\left|g_{\lambda, L}(y)\right|^{2} d y-\frac{4}{3} \pi r^{3} \\
& =\frac{1}{\left\|G_{\lambda, L}\right\|^{2}} \sum_{0<|\zeta|<T} \hat{b}_{x}^{ \pm}(\zeta) e^{-i\left\langle x_{0}, \zeta\right\rangle} \sum_{\substack{\left.|| \xi\right|^{2}-\lambda|<L\\
||\xi-\zeta|^{2}-\lambda \mid<L}} \frac{1}{\left(|\xi|^{2}-\lambda\right)\left(|\xi-\zeta|^{2}-\lambda\right)}+O\left(r^{2} / T\right) .
\end{aligned}
$$

Assume that $\lambda \in \Lambda^{\prime}$, so that $n_{\lambda} \in \widetilde{\mathcal{N}}_{1}$, and proceed as in the proof of Lemma 4.12, recall the bound (4.8), and as before split the first summation (4.9) on the right-hand-side of (4.8) into the sums $\sum^{4}+\sum^{5}$. The bound (4.10) for $\sum^{4}$ still holds:

$$
\sum^{4} \frac{1}{\left(|\xi|^{2}-\lambda\right)^{2}} \ll_{\eta} \lambda^{2 \delta+\eta}
$$

In order to bound $\sum^{5}$, recall that by the bound (4.11) we have

$$
\sum^{5} \frac{1}{\left(|\xi|^{2}-\lambda\right)^{2}} \ll_{\eta}\left\|G_{\lambda, L}\right\|^{2} \frac{L \lambda^{\eta}}{r_{3}\left(n_{\lambda}\right)} .
$$

Write $n_{\lambda}=4^{a} n_{1}$ with $4 \nmid n_{1}$ so that $n_{1}>n_{\lambda} / \log ^{2} n_{\lambda}$. Then by (2.3),

$$
\frac{L \lambda^{\eta}}{r_{3}\left(n_{\lambda}\right)}=\frac{\lambda^{\delta+\eta}}{r_{3}\left(n_{1}\right)} \ll \frac{\lambda^{\delta+\eta}}{n_{1}^{1 / 2-\eta}} \ll \frac{\lambda^{\delta+\eta}}{\left(n_{\lambda} / \log ^{2} n_{\lambda}\right)^{1 / 2-\eta}} \ll \lambda^{\delta-\frac{1}{2}+2.5 \eta} .
$$

The second summation on the right-hand-side of (4.8) satisfies (4.12), and therefore can be bounded similarly. Hence,

$$
\sum_{\substack{\left.|| \xi\right|^{2}-\lambda|<L\\||\xi-\zeta|^{2}-\lambda \mid<L}} \frac{1}{\left(|\xi|^{2}-\lambda\right)\left(|\xi-\zeta|^{2}-\lambda\right)} \ll_{\eta} \lambda^{2 \delta+\eta}+\left\|G_{\lambda, L}\right\|^{2} \lambda^{\delta-\frac{1}{2}+2.5 \eta}
$$


Substituting the bound (4.15) in (4.14), and using property (4) of Lemma 4.4 and the third part of Lemma 4.1, we obtain

$$
\begin{aligned}
\int_{\mathbb{T}^{3}} b_{x}^{ \pm}(y)\left|g_{\lambda, L}(y)\right|^{2} d y-\frac{4}{3} \pi r^{3} & \ll r^{3} T^{3}\left(\frac{\lambda^{2 \delta+\eta}}{\left\|G_{\lambda, L}\right\|^{2}}+\lambda^{\delta-\frac{1}{2}+2.5 \eta}\right)+\frac{r^{2}}{T} \\
& \ll r^{3}\left(T^{3} \lambda^{2 \delta-\frac{1}{2}+2.5 \eta}+\frac{1}{r T}\right) .
\end{aligned}
$$

If $r>\lambda^{-1 / 6+\epsilon}$, and $T=\lambda^{1 / 6-\frac{7}{8} \epsilon}>\frac{1}{r}$, then taking $\eta=\epsilon$, we have

$$
\int_{\mathbb{T}^{3}} b_{x}^{ \pm}(y)\left|g_{\lambda, L}(y)\right|^{2} d y-\frac{4}{3} \pi r^{3} \ll r^{3}\left(\lambda^{2 \delta-\epsilon / 8}+\lambda^{-\epsilon / 8}\right) .
$$

The statement of the lemma follows taking $0<\delta<\epsilon / 16$.

By a standard diagonal argument, and using property (1) of Lemma 4.4, we deduce the following proposition.

Proposition 4.17. There exists a density one subset $\Lambda^{\prime} \subseteq \Lambda$ such that for every $\epsilon>0$

$$
\left.\sup _{\substack{x \in \mathbb{T}^{2} \\ r>\lambda^{-1 / 6+\epsilon}}}\left|\frac{1}{\frac{4}{3} \pi r^{3}} \int_{B_{x}(r)}\right| g_{\lambda, L}(y)\right|^{2} d y-1 \mid \rightarrow 0
$$

as $\lambda \rightarrow \infty$ along $\Lambda^{\prime}$, where $L=\lambda^{\delta}, 0<\delta<\epsilon / 16$.

Proof of Theorem 1.3. By Lemma 4.2, for every $x \in \mathbb{T}^{3}, r>\lambda^{-1 / 6+\epsilon}$, we have

$$
\left.\left|\frac{1}{\frac{4}{3} \pi r^{3}} \int_{B_{x}(r)}\right| g_{\lambda}(y)\right|^{2} d y-1|\leq| \frac{1}{\frac{4}{3} \pi r^{3}} \int_{B_{x}(r)}\left|g_{\lambda, L}(y)\right|^{2} d y-1 \mid+O_{\eta}\left(\lambda^{\eta-\delta / 2}\right)
$$

Hence,

$$
\begin{aligned}
\left.\sup _{\substack{x \in \mathbb{T}^{2} \\
r>\lambda^{-1 / 6+\epsilon}}}\left|\frac{1}{\frac{4}{3} \pi r^{3}} \int_{B_{x}(r)}\right| g_{\lambda}(y)\right|^{2} d y-1 \mid & \leq\left.\sup _{\substack{x \in \mathbb{T}^{2} \\
r>\lambda^{-1 / 6+\epsilon}}}\left|\frac{1}{\frac{4}{3} \pi r^{3}} \int_{B_{x}(r)}\right| g_{\lambda, L}(y)\right|^{2} d y-1 \mid \\
& +O_{\eta}\left(\lambda^{\eta-\delta / 2}\right) .
\end{aligned}
$$

Theorem 1.3 now follows from Proposition 4.17, choosing $\delta<\epsilon / 16$ and $\eta<\delta / 2$.

\section{REFERENCES}

[1] M. V. Berry. Regular and irregular semiclassical wavefunctions. J. Phys. A 10 (1977), no. 12, 2083-2091.

[2] M. V. Berry Semiclassical mechanics of regular and irregular motion. Chaotic behavior of deterministic systems (Les Houches, 1981), 171-271, North-Holland, Amsterdam, 1983.

[3] J. Bourgain, Z. Rudnick. On the geometry of the nodal lines of eigenfunctions of the twodimensional torus. Ann. Henri Poincaré 12 (2011), no. 6, 1027-1053. 
[4] Y. Colin de Verdière. Pseudo-laplaciens. I. Ann. Inst. Fourier (Grenoble) 32 (1982), no. 3, xiii, $275-286$.

[5] Y. Colin de Verdière. Ergodicité et fonctions propres du laplacien. Comm. Math. Phys. 102 (1985), no. 3, 497-502.

[6] M. de Courcy-Ireland. Small-scale equidistribution for random spherical harmonics. Preprint. available online, https://arxiv.org/abs/1711.01317

[7] A. Granville, I. Wigman. Planck-scale mass equidistribution of toral Laplace eigenfunctions. Comm. Math. Phys. 355 (2017), no. 2, 767-802.

[8] X. Han. Small scale quantum ergodicity in negatively curved manifolds. Nonlinearity 28 (2015), no. $9,3263-3288$.

[9] X. Han. Small scale equidistribution of random eigenbases. Comm. Math. Phys. 349 (2017), no. $1,425-440$.

[10] X. Han, M. Tacy. Equidistribution of random waves on small balls. Preprint. available online, https://arxiv.org/abs/1611.05983

[11] G. Harman. On the Erdös-Turán inequality for balls. Acta Arith. 85 (1998), no. 4, 389-396.

[12] H. Hezari, G. Rivière. $L^{p}$ norms, nodal sets, and quantum ergodicity. Adv. Math. 290 (2016), 938-966.

[13] H. Hezari, G. Rivière. Quantitative equidistribution properties of toral eigenfunctions. J. Spectr. Theory 7 (2017), 471-485.

[14] P. Humphries. Equidistribution in shrinking sets and $L^{4}$-norm bounds for automorphic forms. Math. Ann. 371 (2018), 1497-1543

[15] E. Landau. Über die Einteilung der positiven ganzen Zahlen in vier Klassen nach der Mindeszahl der zu ihrer additiven Zusammensetzung erforderlichen Quadrate. Arch. Math. Phys. 13, 305-312 (1908)

[16] W. Z. Luo, P. Sarnak. Quantum ergodicity of eigenfunctions on $P S L_{2}(\mathbb{Z}) \backslash \mathbb{H}^{2}$. Inst. Hautes Études Sci. Publ. Math. (1995), no. 81, 207-237.

[17] M. Young. The quantum unique ergodicity conjecture for thin sets. Adv. Math. 286 (2016), 958-1016.

[18] P. Kurlberg, L. Rosenzweig. Superscars for Arithmetic Toral Point Scatterers. Comm. Math. Phys. 349 (2017), no. 1, 329-360.

[19] P. Kurlberg, H. Ueberschär. Quantum ergodicity for point scatterers on arithmetic tori. Geom. Funct. Anal. 24 (2014), no. 5, 1565-1590.

[20] P. Kurlberg, H. Ueberschär. Superscars in the Šeba billiard. J. Eur. Math. Soc. (JEMS) 19 (2017), no. 10, 2947-2964.

[21] S. Lester, Z. Rudnick. Small scale equidistribution of eigenfunctions on the torus. Comm. Math. Phys. 350 (2017), no. 1, 279-300.

[22] Z. Rudnick, H. Ueberschär. Statistics of wave functions for a point scatterer on the torus. Comm. Math. Phys. 316 (2012), no. 3, 763-782.

[23] P. Šeba. Wave Chaos in Singular Quantum Billiard. Phys. Rev. Let. 64 (1990), no. 16, 18551858.

[24] C. L. Siegel. Über die Classenzahl quadratischer Zahlkörper. Acta Arithmetica 1 (1935), 83-86.

[25] T. Shigehara. Conditions for the appearance of wave chaos in quantum singular systems with a pointlike scatterer. Phys. Rev. E, 50:4357-4370, Dec 1994.

[26] A. I. Shnirel'man. Ergodic properties of eigenfunctions. Uspehi Mat. Nauk 29 (1974), no. 6(180), 181-182. 
[27] H. Ueberschär. Quantum chaos for point scatterers on flat tori. Philos. Trans. R. Soc. Lond. Ser. A. 372 (2014) 20120509.

[28] I. Wigman. N. Yesha, Central limit theorem for Planck scale mass distribution of toral Laplace eigenfunctions. Mathematika, 65 (2019), Issue 3, 643-676.

[29] N. Yesha. Eigenfunction statistics for a point scatterer on a three-dimensional torus. Ann. Henri Poincaré 14 (2013), no. 7, 1801-1836.

[30] N. Yesha. Quantum ergodicity for a point scatterer on the three-dimensional torus. Ann. Henri Poincaré 16 (2015), no. 1, 1-14.

[31] N. Yesha. Uniform distribution of eigenstates on a torus with two point scatterers. J. Spectr. Theory 8 (2018), 1509-1527.

[32] S. Zelditch. Uniform distribution of eigenfunctions on compact hyperbolic surfaces. Duke Math. J. 55 (1987), no. 4, 919-941.

Department of Mathematics, University of Haifa, 3498838 Haifa, Israel

E-mail address: nyesha@univ.haifa.ac.il 